\section{Renin-Inhibitoren - Von 'transition-state' Analogen und Peptidmimetika zu blutdrucksenkenden Wirkstoffen}

\author{
Beat Weidmann*
}

Abstract. During the last 10 years, a great worldwide effort was devoted towards the discovery of orally active renin inhibitors as blood-pressure-lowering drugs. Design principles and synthesis of successive generations of renin inhibitors are discussed using selected examples. The progression from the concept of the 'transition-state' inhibitor, through compounds stable towards protease cleavage and hence towards orally active "nonpeptide' inhibitors is described. All this experience in design, molecular modelling, and synthesis - although not too successful for renin itself - has been applied to the search for inhibitors of other proteolytic enzymes. Thus, the extremely rapid progress made in identifying HIV-protease inhibitors was possible largely through the 'know how' obtained during the search for renin inhibitors.

\section{Einleitung}

\subsection{Das Renin-Angiotensin-System}

Das Renin-Angiotensin-System (RAS) spielt eine zentrale Rolle in der Regulation des Blutdruckes und, damit verbunden, in der Kontrolle des Na-Haushaltes und des Volumengleichgewichtes. Das aktive Prinzip des RAS ist dabei das Angiotensin II (A II), das ein äusserst potentes, blutdruckerhöhendes Octapeptid ist. Die Biosynthese von A II als Endprodukt des RAS ist in Schema 1 dargestellt. Es ist naheliegend, dass Hemmer der Biosynthese dieses endogenen Faktors, blutdrucksenkende Wirkstoffe sein sollten. In der Tat werden Blocker des 'Angiotensin-Converting'-Enzym (ACE) wie Captopril [1] oder Enalapril [2] schon seit Jahren äusserst erfolgreich in der antihypertensiven Therapie eingesetzt. Das grosse Interesse an Blockern des RAS-Systems via Renin-Hemmer kommt von der extrem hohen Spezifität des Renins: Angiotensinogen ist das einzige bis heute bekannte physiologische Substrat. Ganz im Gegensatz zum $\mathrm{ACE}$, das in der Biosynthese von verschiedenen Peptidhormonen involviert ist. Mit der hohen Spezifität des Renins verbindet sich natürlich die Hoffnung, nebenwirkungsfreie Wirkstoffe zu finden. Zudem ist Renin der geschwindigkeitsbestimmende Schritt in der Biosynthese von A II, so dass bei einer Hemmung dieses Enzyms das RAS besonders effizient blockiert werden sollte.

\subsection{Struktur und Mechanismus von Renin}

Renin gehört zur Familie der Aspartylproteasen, die alle viele strukturelle Ge-

*Korrespondenz: Dr. B. Weidmann

Sandoz-Pharma $A G$

Postfach, $\mathrm{CH}-4002$ Basel meinsamkeiten und eine grosse Sequenzhomologie aufweisen. Insbesondere besitzen alle zwei Aspartat-Reste in der aktiven Stelle. Primärsequenz und selbst X-Ray Strukturen sind heute für eine ganze Reihe von AspProteasen inklusive Humanrenin bekannt Fragmenten abgebaut.

Schema 1. Das Renin-Angiotensin-System. Angiotensinogen (mol. wt. ca. 60000), das physiologische Substrat für Renin, wird in der Leber synthetisiert und ist im Blut und anderen extrazellulären Flüssikeiten weit verbreitet. Andererseits ist Renin ein proteolytisches Enzym (mol. wt. ca. 40000) das von einem dafür spezialisierten Teil der Niere (dem sogenannten juxtaglomerulären Apparat) hergestellt, gespeichert und aufgrund von gewissen Stimuli (z.B. niedrigem Blutdruck) in die Blutzirkulation sekretiert wird. Nachdem Angiotensin I durch Renin von seinem Substrat abgespalten worden ist, wird es durch das ACE weiter metabolisiert, das die letzten zwei Aminosäuren des Carboxy-Terminus entfernt. Das entstehende Octapeptid, Angiotensin Il (A II) ist ein äusserst potentes, körpereigenes, blutdruckerhöhendes Prinzip. AII wird durch weitere Enzyme rasch zu biologisch inaktiven

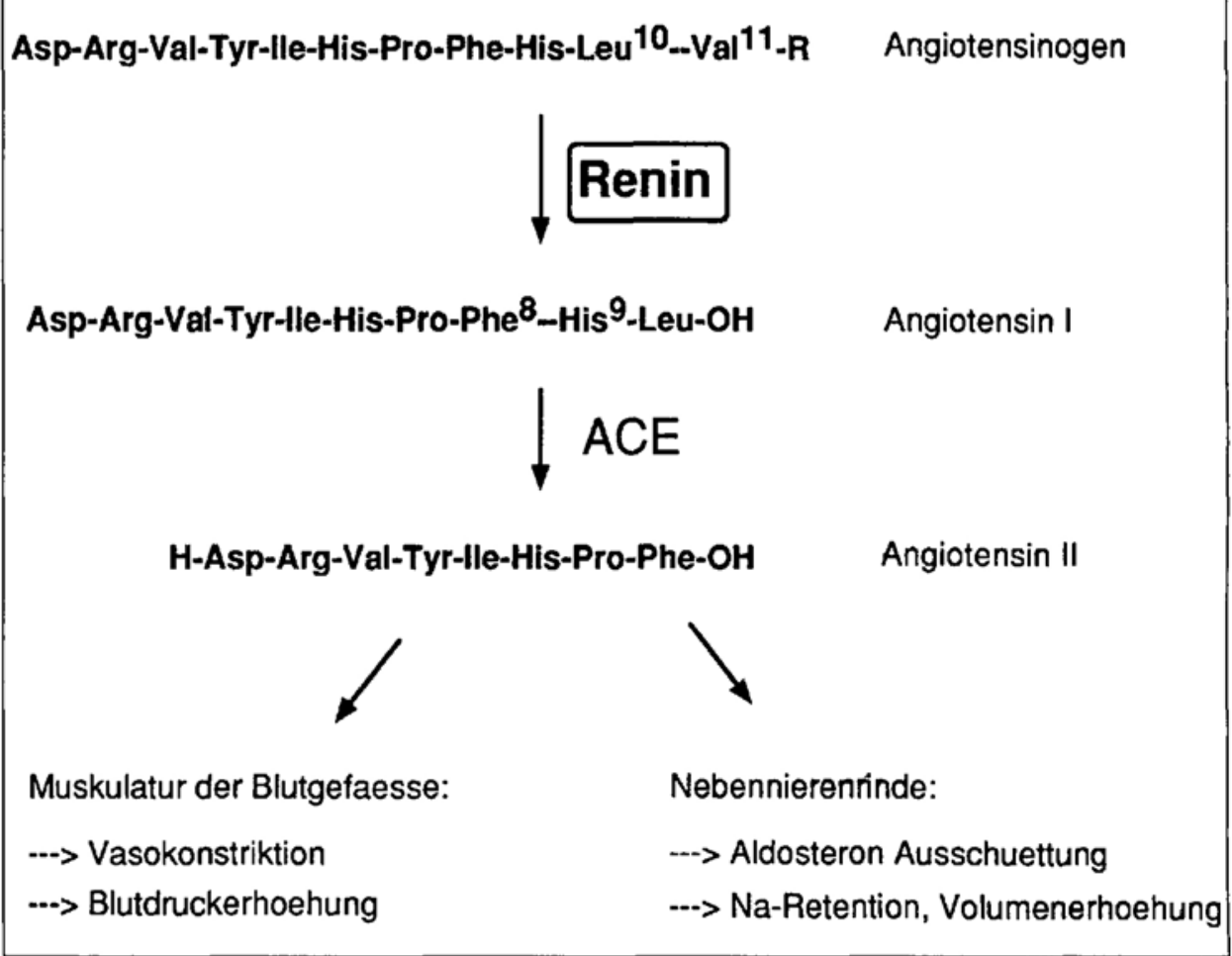




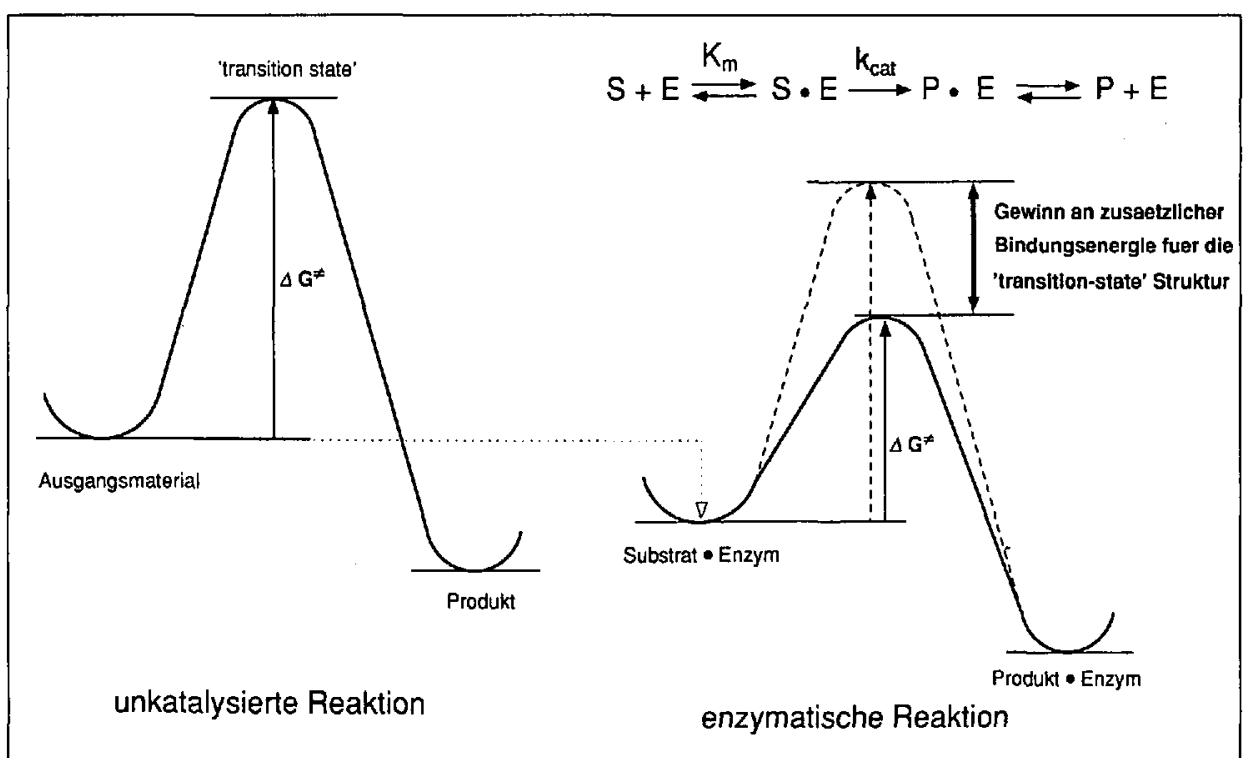

Fig. I. Das Konzept der 'transition-state' Analoga. Dieses schematische Energieprofil verdeutlicht den Zusammenhang zwischen enzymatischer und nichtkatalysierter Reaktion. Die Differenz in der freien Energic zwischen 'transition-state' und Ausgangsmaterial $\Delta G^{\neq}$bestimmt, wie schnell eine Reaktion abläuft. Im enzymkatalysierten Fall ist der eigentlichen Reaktion dic Bildung des Enzym-Substrat-Komplexes vorgelagert. Da sich dieses Gleichgewicht spontan einstellt, liegt der Substrat-Enzym-Komplex auf der Energieskala tiefer. Die Reaktion kann nun aber nicht einfach den gleichen Verlauf wie ohne Enzym beschreiten, weil dies lediglich (wie mil der gestrichelten Linie angedeutet) zu einer Parallelverschiebung des Energieprofiles führen würde. Die für die Reaktionsgeschwindigkeit massgebende Grösse $\Delta G^{\neq}$bliebe dabei unverändert. Ohne auf mechanistische Details einzugehen, kann die beobachtete Beschleunigung der enzyrnatischen Reaktion dadurch erklärt werden, dass das Enzym den Übergangszustand sehr viel besser bindet als das Ausgangsmaterial. Die zusätzliche Bindungsenergie entspricht dabei der Abnahme der Aktivierungsenergie $\Delta G^{\neq}$im Vergleich zur nichtkatalysierten Umsetzung. Oder mit andern Worten: Verbindungen mit der Struktur des Übergangszustandes haben eine besonders hohe Affinität zum Enzym.

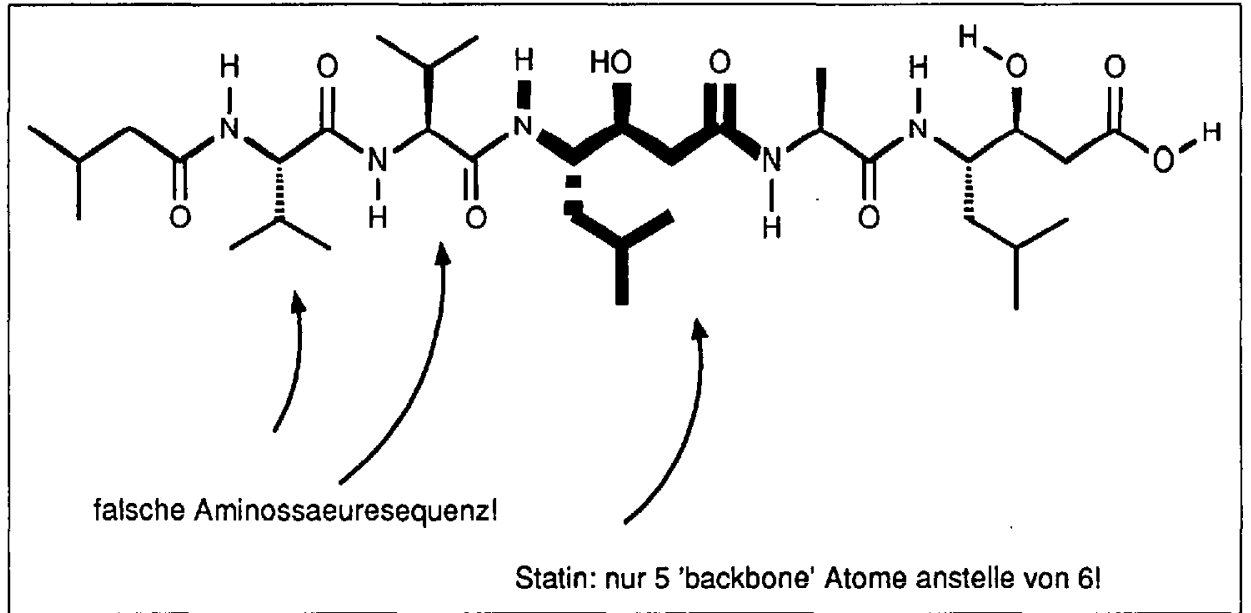

Fig. 2. Pepstatin: Die 'Lead' -Verbindung aus der Natur. Pepstatin enthält die ungewöhnliche Aminosiiure Statin (fett gezeichnet). Sie hat schon von Natur aus eine Struktur, die den aus dem TS-'Approach' abgeleiteten Verbindungen verblüffend ähnlich ist. Dass Pepstatin als solches kein guter Renin-Hemmer ist, kann leicht mit der fehlenden Sequenz-Homologic mit dem Reninsubstrat erklärt werden.
Amid-Bindung an und die Aspartyl-Seitenketten der aktiven Stelle vermitteln die notwendigen Protonentransfers. Eine Zusammenstellung mechanistischer Untersuchungen findet sich in [4].

\section{Das Konzept der 'transition-state' analogen Enzym-Hemmer}

Für den Entwurf von potenten EnzymHemmern gleich welcher Art, hat das sich das Prinzip der 'transition-state'(TS)-Analoga vielfach bewährt und wird im folgenden kurz erläutert: Aus prinzipiellen Gründen kann davon ausgegangen werden, dass die TS-Struktur eine vielfach höhere Affinität zum Enzym haben muss als das Substrat oder die Produkte (vgl. Fig. I), d.h. der Übergangszustand stellt eine ausgezeichnete 'Lead'-Struktur für den Medizinalchemiker dar. Allerdings ist für die meisten Reaktionen die Struktur des TS nicht bekannt und selbst bei einer relativ genauen Vorstellung davon, ist ein Übergangszustand als solcher natürlich nicht herstellbar. Die Kunst besteht darin, Moleküle zu entwerfen, die im Gegesatz zum TS stabil sind, aber trotzdem seine wesentlichen strukturellen Eigenheiten widerspiegeln (vgl. Schema 2). Praktisch wird meist folgendermassen vorgegangen: Es werdén dem TS energetisch naheliegende, kurzlebige Zwischen-Produkte identifiziert, die strukturell noch soweit abgeändert werden, dass stabile, synthetisierbare Analoge resultieren. Diese Verbindungen werden dann anstelle des zu spaltenden Dipeptides in die Substrat-Sequenz eingebaut.

\section{Pepstatin, die 'Lead'-Verbindung aus der Natur}

Obwohl das Konzept der TS-Inhibitoren die Fortschritte auf dem Gebiet der ReninInhibitoren entscheidend beeinflusst hat, muss doch erwähnt werden, dass der Ausganspunkt für einige Arbeitskreise die $\mathrm{Na}$ turstoffchemie war. Umezawa hat schon vor
Schema 2. Planung von TS-Analogen für ProteaseHemmer. Obschon die die genaue Lage des TS bei der Hydrolyse einer Amid-Bindung unbekannt ist, besteht doch wenig Zweifel, dass er entweder kurz vor oder nach der Bildung des tetrahedralen Zwischenprodukt überschritten wird, d.h. dieses kurzlebige Zwischenprodukt kann in erster Näherung als eine gute Approximation des Übergangszustandes angesehen werden. Da aber auch das Z wischenprodukt (ZP) nicht stabil ist muss die Struktur noch weiter abgeändert werden, um zu einem herstellbaren Wirkstoff-Molekül zu kommen. Ein 'Trick' besteht $z$.B. darin, eines oder mehrere der Heteroatome im tetraedrischen $\mathrm{ZP}$ durch C-Atome $\mathrm{Zu}$ ersetzen. So kommt man zur reduzierten Amid-Bindung $A$, dem Hyroxyethyl-Derivat $B$ und, besonders bemerkenswer1, zum hydratisierten Keton C. Bei der Ietzten Struktur erhöhr die $\alpha, \alpha$-Difluoro-Substitution die Elektrophilie in einem Ausmass, dass in wässr. Lsg weitgehend die hyratisierte Form mit der geminalen Diol-Struktur des ZP vorliegt. Es fällt nicht schwer, eine ganze Reihe weiterer Vorschläge zusammenzustellen, die alle mehr oder weniger gute Mimetika für den TS sind.

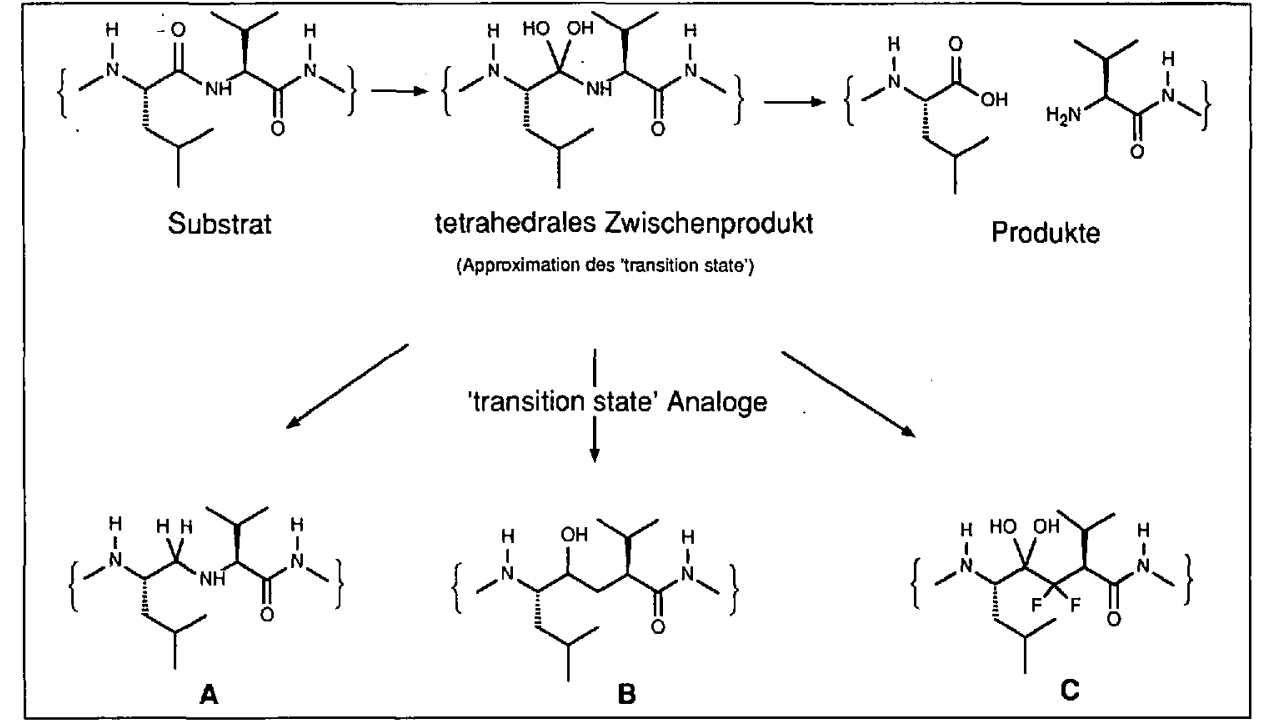


Die erste Generation von 'transition state' - analogen Renin-Hemmnern. Die angegebenen $I C_{50}$-Werte entsprechen der Konzentration der Substanzen, die bei pH 6.0 zu einer $50 \%$ Hemmung von gereinigtem Human-Renin führt, unter Verwendung eines synthetischen Tetradecapeptid als Substrat [34]. Dieser Assay wird von vielen Arbeitsgruppen eingesetzt. Trotzdem sollten jeweils die Originalarbeiten konsultiert werden, wenn $/ C_{50}$-Werte aus verschiedenen Arbeiten miteinander verglichen werden, da die Testbedingungen varieren können. Häufig werden auch Daten für die Hemmung von ungereinigtem Plasma-Renin (also in Gegegenwart von grossen Mengen von Plasmaproteinen) oder, vor allem in älteren Arbeiten, die Hemmung von Mäuserenin angegeben. $K_{\mathrm{D}}$-Werte in dieser Arbeit sind Dissoziationskonstanten bei pH 7.4, bestimmt nach einem bei uns von Evenou et al. entwickelten Bindungsassay [35]. Diese $K_{\mathrm{D}}$-Werte bei einem physiologisch relevantem $\mathrm{pH}$-Wert können bis zu einer Grössenordnung schlechter (d.h. grösser) sein als die in saurem Milieu, dem pH-Optimum für Renin, gemessenen $/ C_{50^{\circ}}$ Werte.

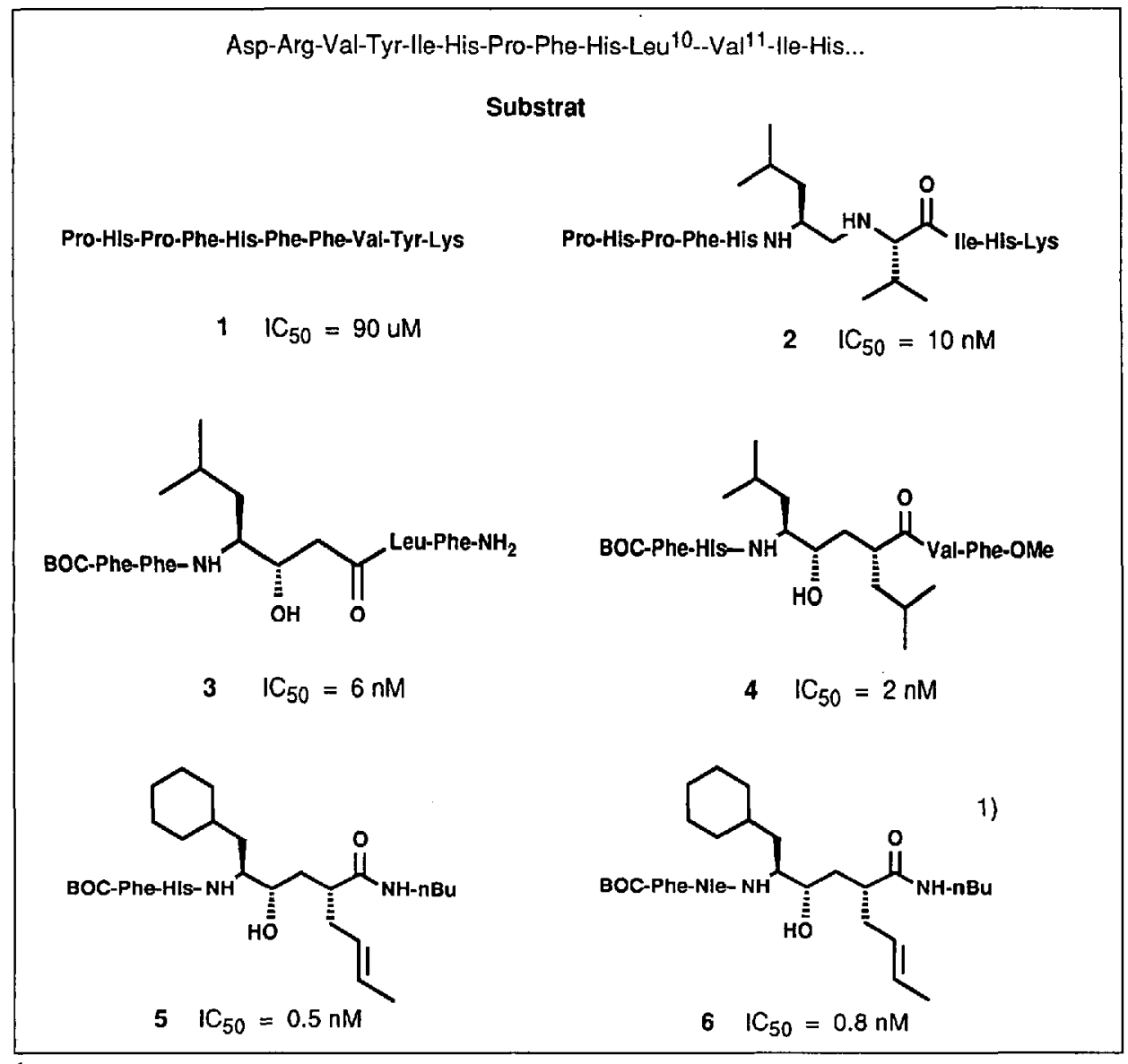

1) Norleucm (Nle) ist (S)-2-Aminohexansïure, ein Isomeres von Leucin. die Erforschung des RAS-Systems, konnte doch mit deren Hilfe in ersten in vivo Experimenten die hypotensive Wirkung von Renin-Hemmern nachgewiesen werden, noch bevor wirksame, kleinmolekulare Hemmer zur Verfügung standen [9].

\section{2. 'Transition-state'-Analoge}

Der Durchbruch zu wirklich potenten und (relativ) kleinmolekularen Hemmern gelang erst Selke et al. mit Verbindungen die ein 'transition-state'-Analogon wie das reduzierte Amidisostere 2 in die SubstratSequenz eingebaut hatten [10]. In rascher Folge wurden dann Verbindungen vom Typ 3 bekannt, die Statin [7] und, als wirkungsvollste Variante davon, das 'Homostatin' [11] (vgl. 4) enthielten. Diese Renin-Hemmer deckten etwa die Bindungsstellen von sechs Aminosäuren des Substrates ab, je drei zu beiden Seiten der zu spaltenden Amid-Bindung zwischen Leu ${ }^{10}$ und Val ${ }^{11}$. Ein weiterer wichtiger Beitrag von Boger et al. [12] war die Entdeckung, dass eine Cyclohexylmethyl-Seitenkette anstelle der substratgerechten (i-Bu)-Gruppe des Leu ${ }^{10}$ die Affinität der daraus gewonnenen Verbindungen drastisch steigern konnte. Diese Verbesserung war so gut, dass das C-terminale Di- oder Tripeptid in älteren Verbindungen durch eine einfache Alkylamino-Gruppe wie z.B. BuNH ${ }_{2}$ ersetzt werden konnte, ohne dabei die nanomolare Dissoziationskonstante zum Renin zu opfern (vgl. 1-4 vs. 5). Ebenfalls wurde schon bald klar, dass auch die restlichen Bindungstellen durch andere Seitenketten, als sie im Substrat vorliegen, besetzt werden können. So werden anstelle von Histidin ${ }^{9}$ auch Phenylalanin, Leucin, Norleucin, $\gamma, \delta-$ ungesättigtes Norleucin und weitere
Jahrzehnten Pepstatin aus Fermentationsbrühen von Actinomyceten isoliert [5]. Es ist ein äusserst potenter Inhibitor $\left(I C_{50}<\right.$ $\left.10^{-9} \mathrm{M} / \mathrm{l}\right)$ für Pepsin, einer Aspartylprotease aus dem Verdaungstrakt. Renin selbst wird von Pepstatin erst im mikromolaren Konzentrationsbereich gehemmt. Ein Vergleich der Struktur von Pepstatin mit dem ReninSubstrat zeigt allerdings schnell, wo Verbesserungen möglich sind (vgl. Fig. 2). Boger et al. konnten in ihrer Pionierarbeit [6] zeigen, dass schon der Ersatz des ValVal-Dipeptides am Aminoterminus durch das substratgerechte Phe-His die Potenz der Hemmer rasch in den nanomolaren Bereich bringt.

\section{Renin-Inhibitoren}

\subsection{Frühe Renin-Inhibitoren}

Die ersten Renin-Inhibitoren mit hoher Affinität zum Enzym waren mono- und polyklonale Antikörper gegen Renin [7] sowie einfache Substrat-Analoge wie 1 [8]. Die Antikörper, obwohl sicher nie als therapeutisch einsetzbare Antihypertensiva gedacht, erwiesen sich als wertvolle Werkzeuge für
Die 'neuen' Hemmprinzipien. Die wenigen Beispiele zeigen die grosse strukturelle Vielfalt, die am C-terminalen Ende 'erlaubt' ist. Nicht immer geben die 'richtigen' Isosteren, d.h. die mit der korrekten Anzahl von 'backbone'Atomen, die besten Hemmer. Vgl. z.B. 7 und 9 mit 8 bzw.10.

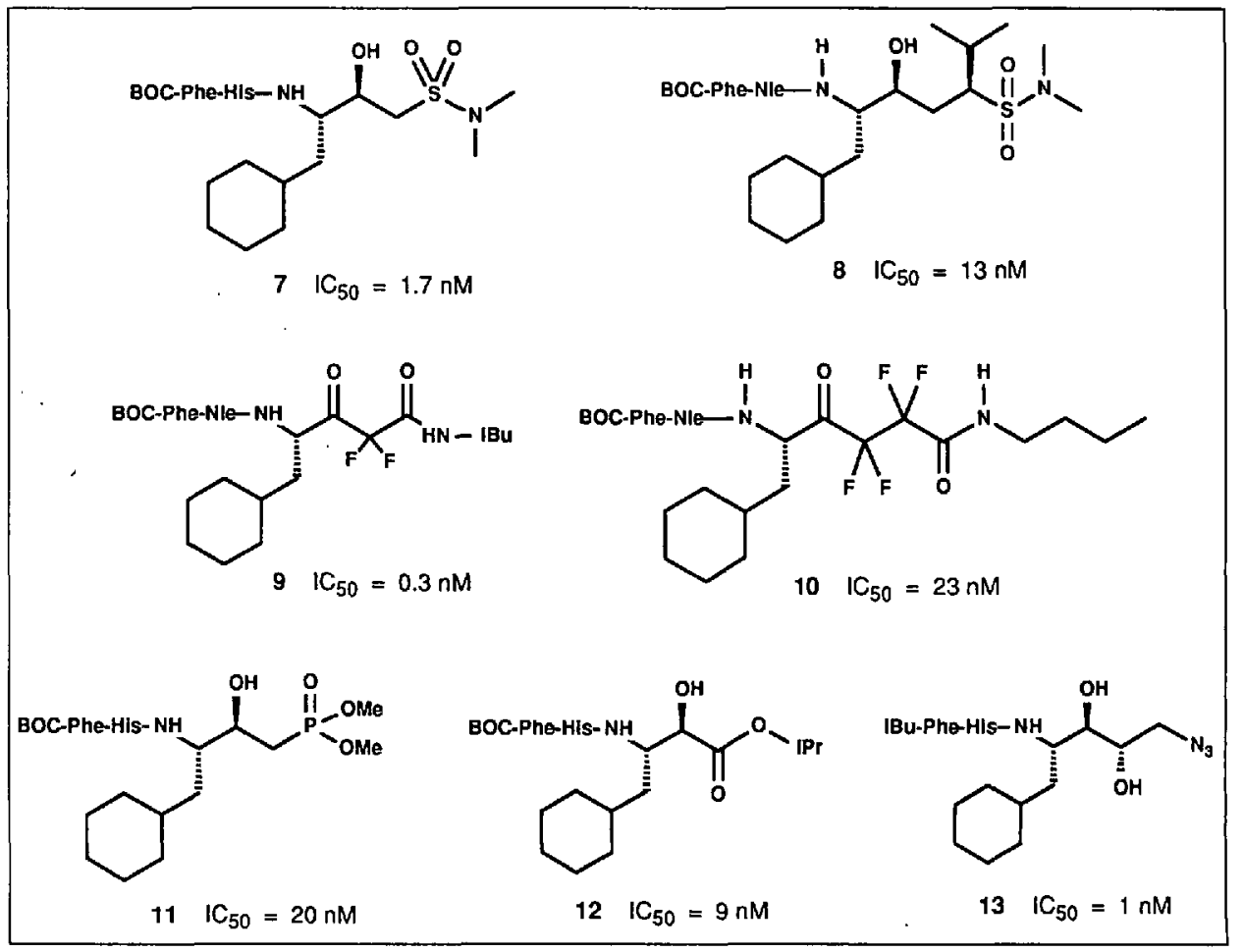


Aminosäuren toleriert (vgl. 3 und 6). Genauso kann die (i-Pr)-Seitenkette von $\mathrm{Va}$ lin ${ }^{11}$ durch eine ganze Reihe von bevorzugt hydrophoben Substituenten wie $\mathrm{Me}, \mathrm{Pr}, \mathrm{Bu}$, Butenyl, Ph etc. ersetzt werden (vgl. 4-6 vs. 2). Wie weiter unten beschrieben (Kap.4.5), hat diese überraschend grosse Toleranz des Renins bei der Planung von nichtpeptidischen Hemmern eine wichtige Rolle gespielt.

Diese raschen Fortschritte zeigten einerseits eindrücklich wie erfolgreich das Konzept der 'transition-state'-analogen EnzymHemmer eingesetzt werden konnte. Andererseits bearbeiteten, ausgehend von diesem Konzept, praktisch alle Arbeitsgruppen auf diesem Gebiet (viele davon naturgemäss in der Pharmaindustrie) die gleichen, oben er- wähnten 'Lead'-Verbindungen. Wohl nicht zuletzt deshalb wurden in der Folge Dutzende von neuen TS-Analogen insbesondere auch in der Patentliteratur beschrieben [13]. Eine kleine Auswahl sind 7-13. Es fällt auf, dass praktisch alle Verbindungen die erwähnte Cyclohexylmethyl-Seitenkette aufweisen und am C-terminalen Ende, das für eine starke Bindung am Renin offenbar weniger wichtig ist, modifiziert sind.

Der Optimismus, der durch die schnellen Fortschritte in Planung und Synthese von äusserst potenten und dabei hochselektiven Hemmern geweckt wurde, machte in allen Labors grosser Emüchterung Platz, sobald die neuen Verbindungen auf ihre Wirkung in vivo untersucht wurden. Diese erste Generation von TS-Renin-Hemmer waren nicht nur sehr schwer wasserlöslich und damit für die Pharmakologie schwierig handhabbar, sondem zeigte kaum eine der beschriebenen Verbindungen eine signifikante Wirkung, wenn sie oral verabreicht wurde (Aktivität bei oraler Applikation ist für einen antihypertensiven Wirkstoff fast eine Grundvoraussetzung!). Intravenös appliziert hemmten viele Verbindungen zwar die Plasma-Renin-Aktivität (PRA) und senkten den Blutdruck, die Halbwertszeiten dieser Effekte betrug aber oft nur wenige min. All diese negativen Eigenschaften wurden mit der peptidartigen Natur dieser Substanzen in Verbindung gebracht. Es hat in der Folge nicht an Anstrengungen gefehlt, diese Probleme durch entsprechende strukturelle Modifikationen zu lösen.
Wasserlösliche Reninhemmer. Nur drei Beispiele anstelle der vielen Dutzend in [36] beschriebenen, hydrophilen Modifikationen des $N$-wie auch des $C$-Terminales. Die grosse Variabilität, die hier möglich ist qhne einen wesentlichen Einfluss auf die Bindung ans Renin zu haben, lässt den Schluss zu dass diese Stellen schon praktisch ausserhalb der 'activ site' au liegen kommen.
Chymotrypsin resistente ReninHemmer. Wie die Beispiele zeigen, ist es relativ einfach, gegen Chymotrypsin resistente, aber dabei doch sehr potente ReninHemmer zu entwerfen. Der Grund liegt darin, dass die Modifikationen rund um den kritischen Phenylalanin-Rest bei Chymotrypsin unmittelbar vor derzu spaltenden Amidbindung zu liegen kommen (der sogenannten $\mathrm{P}_{1}$-Bindungsstelle). Bei Renin kommt die modifizierte Aminosäure aber an die $\mathrm{P}_{3}$ Bindungstelle zu liegen, also viel weiter weg von der 'active site', wo die strukturellen Bedingungen für eine gute Bindung offenbar weniger genau definiert sind.

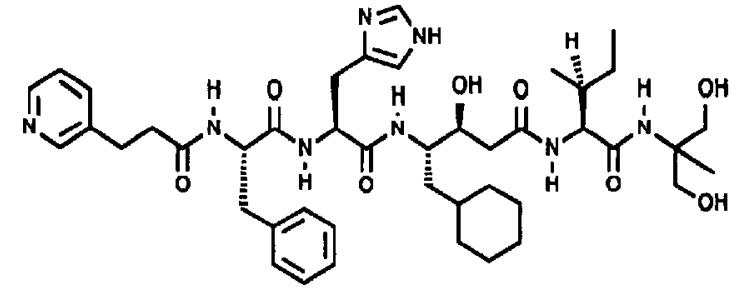

$15 K_{D}=1.0 \mathrm{nM}$

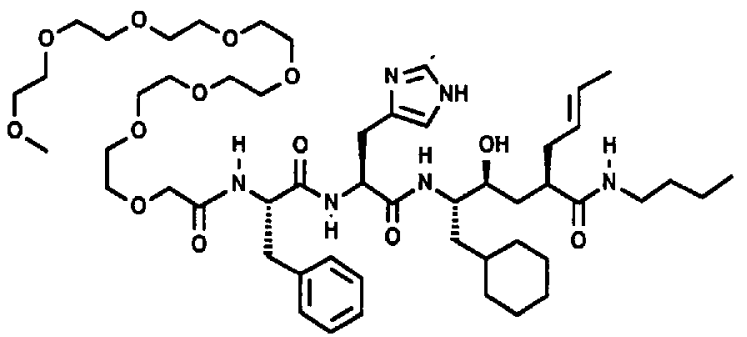

SDZ 213-837 $K_{D}=0.5 n M$

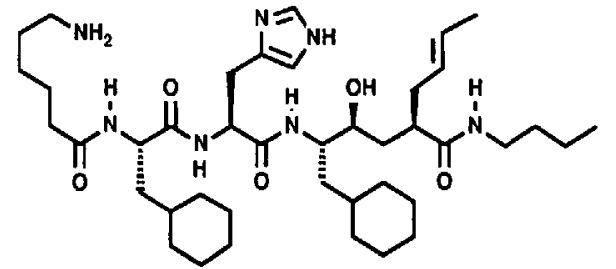

$14 K_{D}=0.6 n M$<smiles></smiles>

SDZ 214-555 $K_{D}=2 n M$

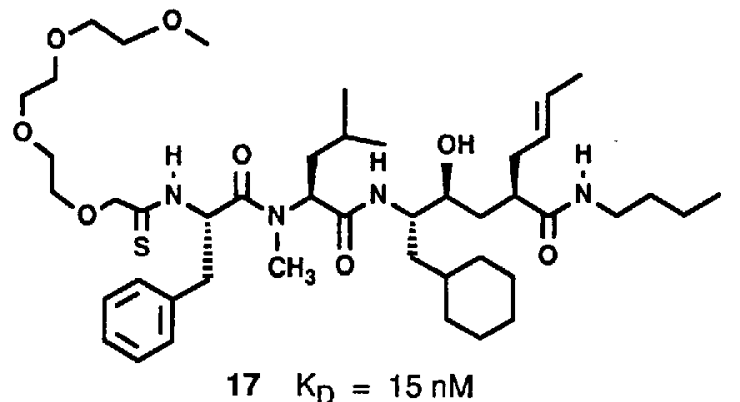<smiles>COc1ccc(C[C@H](NC(=O)N2CCOCC2)C(=O)N[C@@H](Cc2cnc[nH]2)C(=O)N[C@@H](CC2CCCCC2)[C@@H](O)[C@@H](O)CC(C)C)cc1</smiles>

$16 I_{50}=0.8 \mathrm{nM}$<smiles>CC(C)C[C@H](O)[C@H](O)[C@H](CC1CCCCC1)NC(=O)[C@H](Cc1cn[nH]c1)NC(=O)[C@H](Cc1ccccc1)OC(=O)N1CCOCC1</smiles>

$18 \quad \mathrm{C}_{50}=0.8 \mathrm{nM}$ 
CHIMIA 4.5 (1991) Nr. 12 (Dezentrer)

\subsection{Wasserlösliche Renin-Hemmer}

Aspartylproteasen spalten Peptid-Bindungen bevorzugt zwischen hydrophoben Aminosäuren; Human-Renin zwischen Leucin und Valin. Zwangsläufig erhält man mit TS- und Substratanalogen Hemmern auch stark hydrophobe Moleküle. Es hat sich jedoch gezeigt, dass sich sowohl das $\mathrm{C}$ wie auch das N-terminale Ende der Blocker durch hydrophile Reste modifizieren lassen, ohne dabei die Affinität zum Enzym zu kompromittieren (vgl. 14, 15 und SDZ 213837). Viele der so erhaltenen Substanzen sind, im Gegensatz zu älteren Verbindungen, ohne weitere Hilfsmittel in $\mathrm{H}_{2} \mathrm{O}$ bei physiologischen $\mathrm{pH}-$ Werten löslich. Leider wurde die oben erwähnte ungünstige Pharmakokinetik durch die veränderten physikochemischen Eigenschaften nur teilweise verbessert. Insbesondere konnte mit kaum einem dieser hydrophilen Hemmer eine signifikannte orale Aktivität nachgewiesen werden.

\subsection{Chymotrypsin-resistente Renin- Hemmer}

Die Suche nach den Gründen für die schlechte 'Bioverfügbarkeit' hat schon bald einen gemeinsamen Schwachpunkt aller Renin-Hemmer zu Tage gefördert [14]: Die 'traditionellen' Renin-Hemmer enthielten praktisch immer Dipeptide wie Phe-His oder Phe-Nle als Bausteine (vgl. 1-6 und 7-13) Es überrascht eigentlich nicht, dass diese Phenylalanin enthaltenden Peptide gute Substrate für $\alpha$-Chymotrypsin sind, einer wichtigen Protease des Verdaungstraktes (Schema 3). Diese rasche Abbaubarkeit ist in Zusammenhang mit der schlechten oralen Aktivität gebracht worden. Konsequenterweise wurde darauf eine ganze Generation von Chymotrypsin-resistenten Renin-Hemmer synthetisiert. Es genügte, das Phenyla$\operatorname{lanin}^{8}$ in der Substrat-Sequenz durch ein geeignetes 'Bio-Isosteres' zu ersetzen. Beispiele sind (Trimethylsilyl)alanin [15] und $O$-Methyltyrosin [16]. Auch die N-Methylierung der Amid-Bindung zwischen PheHis oder Phe-Nle oder der Ersatz der Amino-Gruppe des Phenylalanins [17] ergaben gegen $\alpha$-Chymotrypsin weitgehend stabile, aber immer noch sehr potente Renin-Hemmer (vgl. SDZ 214-555 und 16-18).

Diese Verbindungen waren weitgehend gegen den Abbau durch proteolytische Enzyme geschützt, weil auch das $\mathrm{C}$ - und $\mathrm{N}$ -

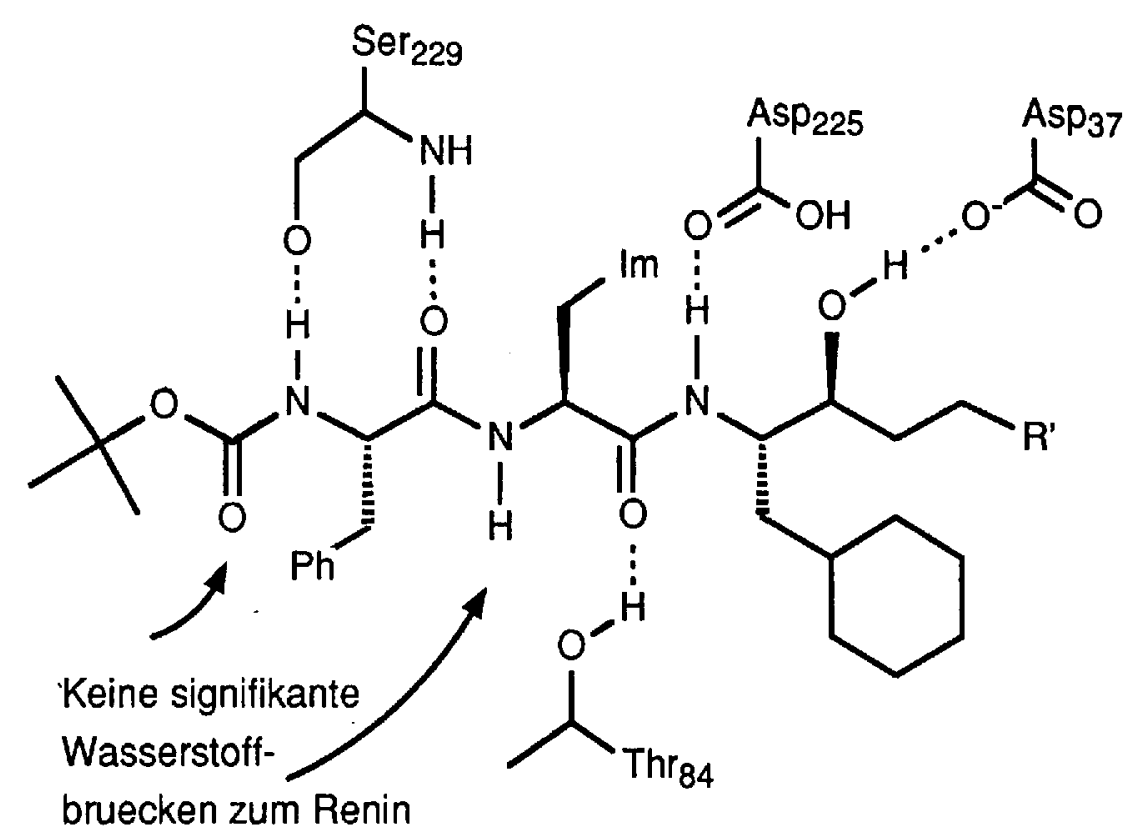

Fig. 3. Wechselwirkung eines Renin-Hemmers mit Renin. Vedani's YETY-Programm [18] ist besonders geeignet um ein Netz von H-Brückenbindungen zu optimieren. Es berücksichtigt nicht nur deren Länge, sondern als Exklusivität auch dic ausgeprägte Richtungsabhängigkeit dieser Art von Wechselwirkungen. Natiirlich werden gleichzeitig auch die Van-der-Waals-Interaktionen optimiert

terminale Ende derivatisiert und damit dem 'Zugriff' von Exopeptidasen entzogen war. Intravenös verabreicht zeigten viele dieser Hemmer wie z.B. SDZ 214-555 eine ausgeprägte und langanhaltende Blutdrucksenkung sowie eine vollständige Hemmung der PRA. Die Entäuschung war (wohl nicht nur in unserer Arbeitsgruppe) gross, dass trotz diesen weiteren Verbesserungen immer noch praktisch keine orale Aktivität nachzuweisen war. Es setzte sich immer mehr die Ansicht durch, dass nicht nur die metabolische Instabilität eine grosse Hürde für die orale Wirksamkeit ist, sondern dass der peptidische Charakter per se einer effektiven Resorption aus dem (Magen/Darm)Trakt im Wege steht, auch wenn (biochemisch) völlig stabile Wirkstoff-Moleküle vorliegen. Gesucht waren also Strukturvorschläge die zu nichtpeptidischen ReninHemmern führten.

\subsection{Nichtpeptidische Hemmer}

Für den rationalen Entwurf von nichtpeptidischen Hemmern, also von Verbindungen, die keine $\alpha$-Aminosäuren mehr enthalten, standen bei uns am Anfang 'molecular-modelling'-Studien über die Art der

Schema 3. Inaktivierung eines Renin-Hemmers durch $\alpha$-Chymotrypsin. SDZ 213-837 wurde in wenig MeOH gelöst und zu einer gepufferten Lsg. von $1 \mathrm{mg} \alpha$-Chymotrypsin/ml (von Sigma) gegeben. Nach $30^{\prime}$ wurde das Enzym mit Aceton gefällt, der Überstand filtriert und eingedampft. Im Rückstand konnte dirch MS keine Spur mehr von 213-837 mehr nachgewiesen werden. Statt dessen liegen nur mehr die angegebenen Spaltprodukte vor.

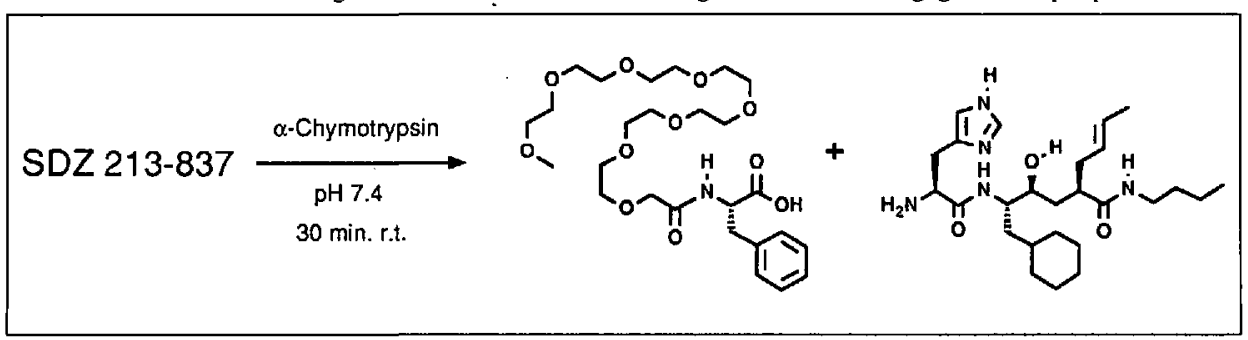

Wechselwirkung der peptidischen Hemmer mit dem Enzym im Vordergrund. Für das Renin wurde dabei eine aufgrund der XRay-Koordinaten von Rhizopuspepsin modellierte Struktur verwendet. Die Inhibitoren wurden mit dem SYBYL-Programm modelliert und am Bildschirm manuell in die 'active site' des Renin-Modelles eingepasst. Jeder Komplex wurde anschliessend durch das Molecular-Mechanics-Program YETY [18] optimiert. Das Ergebnis dieser Arbeiten ist schematisch in Fig. 3 wiedergegeben [19]. Weder für die N-Acyl-Gruppe des Phenylalanins ${ }^{8}$ noch für die $\mathrm{NH}-$ Gruppe der Amid-Bindung zwischen Phe ${ }^{8}$ und $\mathrm{His}^{9}$ konnten eine Interaktion mit dem Enzym gefunden werden. Unsere Bemühungen konzentrierten sich deshalb vor allem darauf, Dipeptid-Mimetika zu finden, in welchen diese Amid-Bindung durch ein entsprechendes Isosteres ersetzt ist. Die 'molecular-modelling'-Hypothesen konnten voll bestätigt werden. Ketomethylen-, Sulfomethylen- und Hydroxyethylen-Isostere - in allen ist die im Substrat vorhandene NHGruppe durch eine $\mathrm{CH}_{2}$-Gruppe ersetzt ergeben in Kombination mit einem TSAnalogon Renin-Hemmer mit nanomolarer Affinität, ohne eine einzige $\alpha$-Aminosäure!

Auch die $N$-Acylgruppe des Phenylalanins kann ersetzt (SDZ 214-910) oder verschoben (SDZ 217-193) werden, ohne dass die Affinität zu Renin wesentlich vermindert wird. Weiter haben wir uns die schon oben beschriebene Struktur-Aktivitätsbeziehung zu Nutze gemacht, und aus synthetischen Gründen vorerst gar nicht versucht, ein Phe-His Isosteres mit den 'korrekten' Seitenketten herzustellen [20]. Statt dessen liegt den Hemmern 214-910 und 217-172 eine, bis auf die Schutzgruppen und den Oxidationszustand, translationssymmetri- 
'Nichpeptidische' ReninHemmer. Wie erwähnt fällt bei den Hemmern SDZ 214-910 und SDZ 217-172 die translationssymmetrische Struktur auf. SDZ 217-172 ist im Gegensatz zu den weiter oben stehenden gut wasserlöslich und oral eine der aktivsten Verbindungen, die wir je geprüft haben. In Fig. 4 sind die Daten der Testierung dieser Substanz an wachen Totenkopfaffchen festgehalten. Interessint ist die Entstehungsgeschichte von $/ C l$ 's ReninHemmer 20 [37]. Eine grössere Anzahl Arylessigsäuren wurden mit cinem TS-Analogen kombiniert. Die beste Verbindung aus diesem 'semirandom Scree-ning' wurde dann weiter zur Verbindung 20 optimiert. CGP 38560 war einer der ersten Renin-Hemmer, die nur noch Histidin als einzige $\alpha$-Aminosäure enthielten und gehört zu den am besten untersuchten Hemmern, über die berichtet wurde. Sogar klinische Studien wurden damit durchgeführt, wenn auch mit entäuschenden Resultaten [39].

Fig. 4. In-vivo Aktivität von SDZ 217-172. Dosen von 0.15 $\mathrm{mg} / \mathrm{kg}$ (intravenös) oder $3 \mathrm{mg} /$ $\mathrm{kg}$ (per oral) wurden wachen Totenkopfaffchen verabreicht, die mit dem Diuretikum Furosemide $(50 \mathrm{mg} / \mathrm{kg}$ ) vorbehandelt wurden. PRA und systolischer Blutdruck (SBP) wurden wïhrend $5 \mathrm{~h}$ verfolgt und sind im Diagramm als prozentuale Änderung zum Ausgangswert aufgetragen. Nur Tiere mit einer $P R A$ von $\geq 10 \mathrm{ng} A l 1 / \mathrm{m} / \mathrm{h}$ (nach Furosemide-Behandlung) wurden für die Auswertung berücksichtigt. Man beachte die im späteren Versuchsverlauf auftretende Diskrepanz zwischen PRA und Blutdrucksenkung.
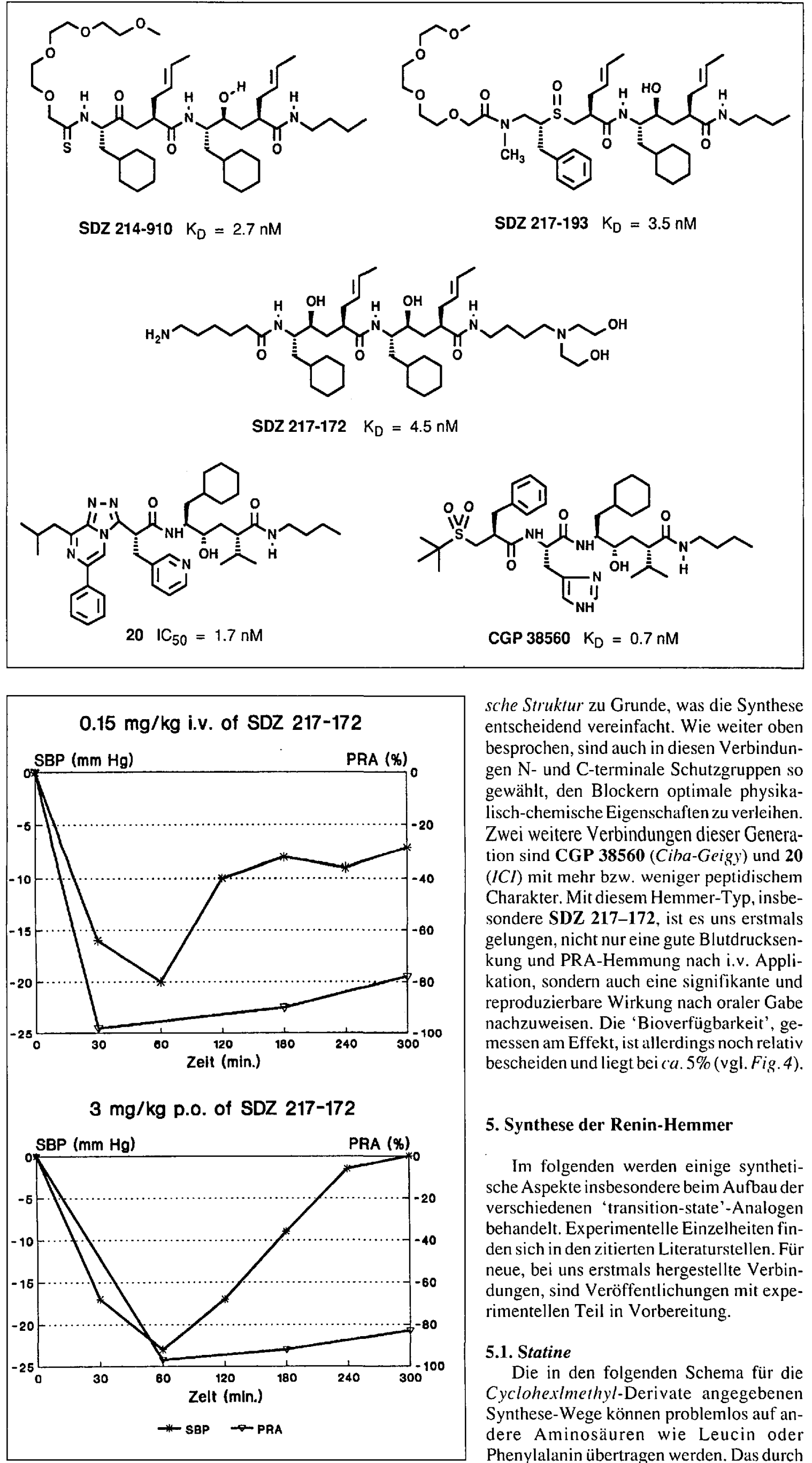

sche Struktur zu Grunde, was die Synthese entscheidend vereinfacht. Wie weiter oben besprochen, sind auch in diesen Verbindungen N- und C-terminale Schutzgruppen so gewählt, den Blockern optimale physikalisch-chemische Eigenschaften zu verleihen. Zwei weitere Verbindungen dieser Generation sind CGP 38560 (Ciha-Geigy) und 20 (ICI) mit mehr bzw. weniger peptidischem Charakter. Mit diesem Hemmer-Typ, insbesondere SDZ 217-172, ist es uns erstmals gelungen, nicht nur eine gute Blutdrucksenkung und PRA-Hemmung nach i.v. Applikation, sondern auch eine signifikante und reproduzierbare Wirkung nach oraler Gabe nachzuweisen. Die 'Bioverfügbarkeir', gemessen am Effekt, ist allerdings noch relativ bescheiden und liegt bei c $a .5 \%$ (vgl.Fig.4).

\section{Synthese der Renin-Hemmer}

Im folgenden werden einige synthetische Aspekte insbesondere beim Aufbau der verschiedenen 'transition-state'-Analogen behandelt. Experimentelle Einzelheiten finden sich in den zitierten Literaturstellen. Für neue, bei uns erstmals hergestellte Verbindungen, sind Veröffentlichungen mit experimentellen Teil in Vorbereitung.

\subsection{Statine}

Die in den folgenden Schema für die Cyclohex/methyl-Derivate angegebenen Synthese-Wege können problemlos auf andere Aminosäuren wie Leucin oder Phenylalanin übertragen werden. Das durch 
katalytische Hydrierung (Rh auf Alox) von $\mathrm{N}$-Boc-Phenylalanin leicht zugängliche $\mathrm{N}$ Boc-Cyclohexylalanin wird mit 3,5-Dimethylpyrazol und Dicylohexylcarbodiimid (DCC) ins entsprechende Pyrazolid übergeführt. Reduktion mit Diisobutylaluminiumhydrid (DIBAH) und vorsichtige Hydrolyse mit verdünnter $\mathrm{HCl}$ führt selektiv zum $N$ Boc-Aminoaldehyd, der wegen Racemisierungsgefahrohne weitere Reinigung mit dem Lithium-enolat von Essig-ester umgesetzt wird. Das daraus resultierende (2:1)-Diastereoisomerengemisch der geschützten Statine kann durch Chromatographie an Kieselgel leicht getrennt werden. Wie in Schema 4 angegeben, können diese Zwischenprodukte in einfacher Weise in eine geeignete Substratsequenz eingebaut werden: Aminolyse des Esters mit einem geeigneten Amin, Abspaltung der BocSchutzgruppe mit $\mathrm{CF}_{3} \mathrm{COOH}$ und Acylierung der freien Amino-Gruppe mit dem gewünschen Dipeptid (oder Dipeptid-Ersatz) z.B. Boc-Phe-Nle-OH unter Verwendung von DCC und Hydroxybenzotriazol (HOBT) [21]. Unter diesen Bedingungen wird keine oder, bei Histidin enthaltenden Peptiden, nur eine sehr geringe $(\leq 5 \%)$ Racemisierung der aktivierten Aminosäure beobachtet.

\subsection{Difluorstatone}

Die Herstellung der Difluorstatone (Schema 5) folgt im Prinzip den nichthalogenierten Verbindungen. Anstelle des Lithium-enolates von Essig-ester wird jedoch das Reformatzky-Reagens aus Bromodifluoroessigsäure-ethylester und aktiviertem $\mathrm{Zn}$ in siedendem THF eingesetzt [22]. Interessanterweise entsteht in diesem Fall praktisch nur ein Diastereoisomeres. Der Difluoroalkohol wird auf die gleiche Art wie in Schema 4 in die gewünschte Substrat-Sequenz eingebaut. Zuletzt erfolgt die nicht immer leichte Oxidation des Statins zum Staton. Besonders bewährt haben sich dabei Collin's Reagens, $\mathrm{CrO}_{3}$.2Pyridin [23], DMSO aktiviert mit DCC/Cl ${ }_{2} \mathrm{CHCOOH}$ [24] oder Dess Martin's Reagens [25].

\subsection{Hydroxyethylen Isostere ('Homosta- tine') \\ Eine gute Übersicht über die zahlreichen} bis jetzt beschriebenen Synthesen von $\mathrm{Hy}$ droxyethylen-Isosteren findet sich in [26]. Wir haben den in Schema 6 beschriebenen Weg gewählt und damit problemlos $100 \mathrm{~g}$ Mengen der entsprechenden Verbindungen herstellen können. Das schon in Schema 4 erwähnte $N$-Boc-Cyclohexylmethylalaninal wird mit dem Dilithium-Derivat von Propiolsäure als Homoenolat-Äquivalent [27] umgesetzt. Hydrierung des Adduktes und säurekatalysierte Cyclisierung liefert das Lacton als (3:1)-Diastereoisomerengemisch zugunsten des gewünschten (4S)-Isomeren. Dieses kann mit Lithium-diisopropylamid (LDA) ins Dianion übergeführt werden, das mit ausgezeichneter trans-Diastereoselektivität (>95:5), mit z.B. mit Crotyl-bromid, alkyliert werden kann. Allerdings ist kom-
Schema 4

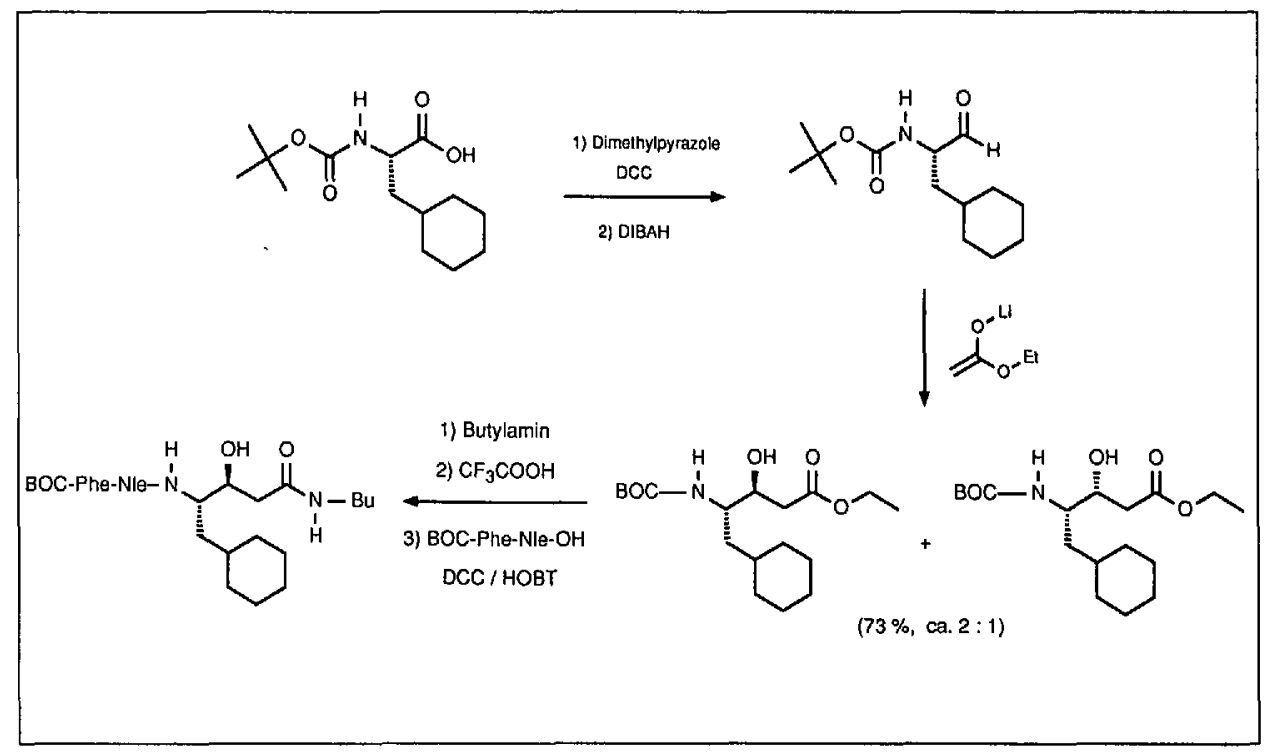

Schema 5<smiles>CC(C)(C)OC(=O)N[C@@H](C=O)CC1CCCCC1</smiles>

$\mathrm{Br}-\mathrm{CF}_{2} \mathrm{COOEt}$<smiles>CCOC(=O)C(F)(F)[C@@H](O)[C@H](CC1CCCCC1)NC(=O)OC(C)(C)C</smiles>

(85\%, ein Isomeres) 1) l-Butylamin
2) $\mathrm{CF}_{3} \mathrm{COOH}$
3) $\mathrm{BOC}-\mathrm{Ph} \theta-\mathrm{NleOH}$
$\mathrm{DCC} / \mathrm{HOBT}$
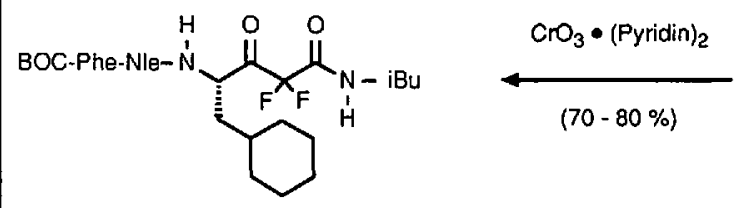
BOC-Ph日-Nle-N)

Schema 6<smiles>CC(C)(C)OC(=O)N[C@@H](C=O)CC1CCCCC1</smiles>

1) Li-C=C-COOLi

$(50 \%)$<smiles>CC(C)(C)OC(=O)N[C@H](CC1CCCCC1)[C@@H](O)C#CC(=O)O</smiles><smiles></smiles>

1) LDA

2) Crotylbromid

$(70 \%)$

1) $\mathrm{H}_{2} / \mathrm{Pd}-\mathrm{C}$ 2) $\mathrm{H}^{+}$

(>95:5) 
Schema 7

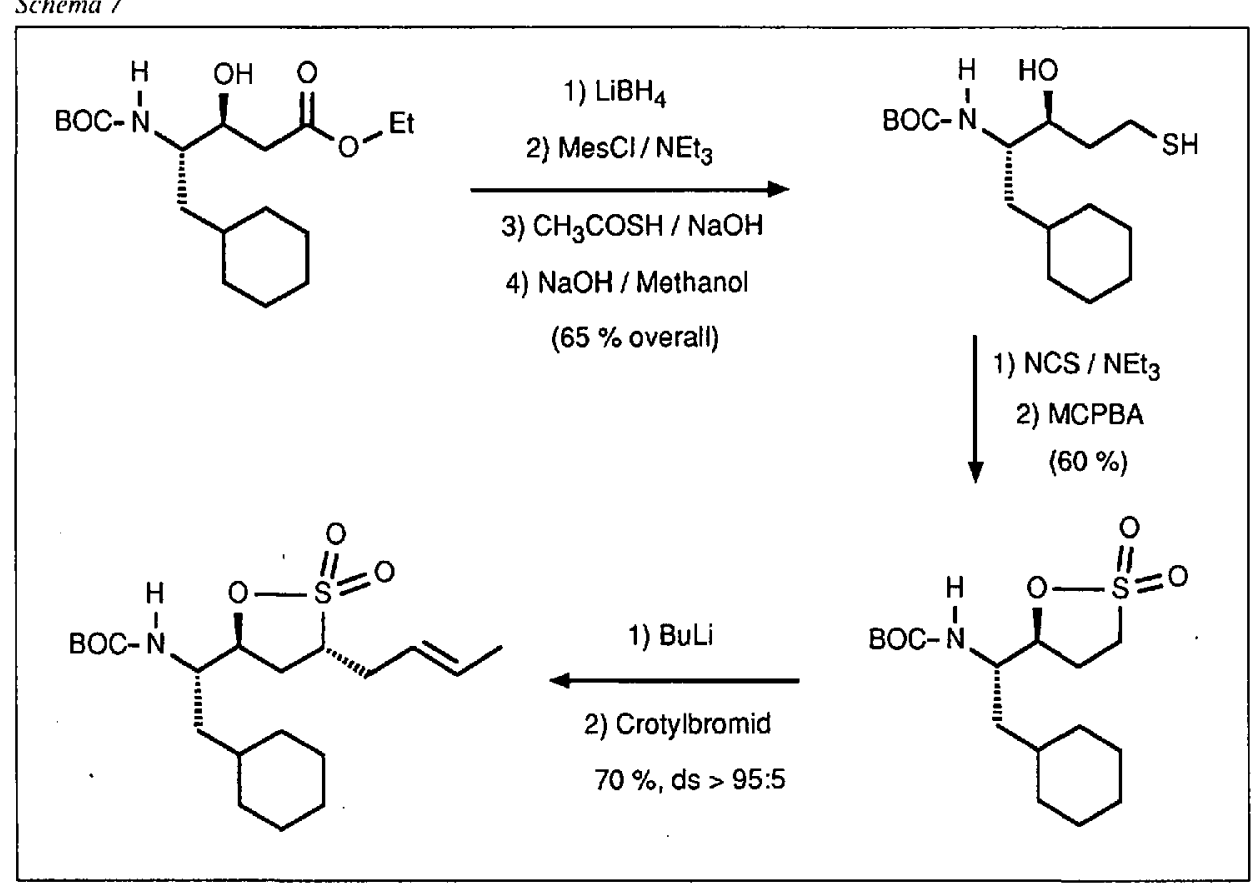

merziell erhältliches $(E)$-Crotyl-bromid mit $10-20 \%$ des $(Z)$-Isomeren verunreinigt. Ein bezüglich der Konfiguration der $(\mathrm{C}=\mathrm{C})$ Bindung einheitliches Produkt wird mit $(E)$ Crotyl-triflat erhalten, das sich in situ aus Crotyl-alkohol, BuLi und Trifluoromethansulfonsäure-anhydrid erzeugen lässt. Ein Nachteil dieses Synthese-Weges ist, dass nur Seitenketten eingeführt werden können, die sich von reaktiven Alkylierungsmittel wie Mel oder Allyl- und Benzyl-halogeniden ableiten. Der Einbau in eine Substrat-Sequenz erfolgt dann genau gleich wie im Schema 4 beschrieben.

5.4. Sulfonsäure-Analoge der $H y$ droxyethyl-Isosteren ('Sulfohomostatine')

Zur Synthese dieses Sulfonsäure-Analogons (Schema 7) der Homostatine kann das nach Schema 4 hergestellte Cyclohexlmethyl-'statin' verwendet werden. Reduktion der Ester-Gruppe, selektive Mesylierung und Substitution der primären $\mathrm{OH}$-Gruppe durch das Na-Salz der Thioessigsäure liefert nach basischer Abspaltung der Ac-Gruppe stereochemisch einheitliches Hydroxythiol. Letzteres kann mit $N$-Chlorosuccinimid (NCS) unter basischen Bedingungen zum Sulfensäure-ester cyclisiert und mit $m$ Chloroperbenzoesäure (MCPBA) weiter zum Sulfonsäure-ester oxidiert werden. Die weiteren Stufen entsprechen genau dem in Schema 6 beschriebenen Verfahren. Das Dilithio-Derivat kann jedoch direkt mit BuLi (anstelle von LDA) hergestellt werden.

\subsection{Tetrafluoro-ketomethylen-Isostere} (Tetrafluoro-'Homostatone')

Die in Schema 8 beschriebene elektrochemische Synthese unter Verwendung des in Schema 5 beschriebenen $N$-Boc-Difluorostatins als Kupplungskomponente in einer gemischten Kolbe-Elektrolyse verläuft zwar nur mit sehr mässiger Ausbeute [28]. Die Kürze des Verfahrens stellt aber im

\section{Im Gegensatz zu den von Anfang an ergiebigen chemischen und biochemischen Ansätzen für die Entwicklung eines Renin- \\ 6. In-vivo-Aktivität von Renin- \\ Inhibitoren}

Schema 8
Hemmers hatte die Pharmakologie einen ungleich schwereren Stand. Die Probleme begannen schon bei der Suche nach einem geeigneten Tiermodell: Es hatte sich gezeigt, dass z.B. Ratten-Renin durch viele für $\mathrm{Hu}$ man-Renin optimierte Hemmer nur noch wenig oder gar nicht mehr gehemmt wird. Das bedeutete, dass bei diesen, von Kreislaufpharmakologen bevorzugten Tieren, der Einfluss eines Renin-Hemmers auf den Blutdruck nur bei gleichzeitiger Infusion von Human-Renin bestimmt werden konnte. Reines Human-Renin war aber, bevor gentechnologisch hergestelltes zur Verfügung stand, nur in sehr beschränkten Mengen zugänglich. Viele Arbeitsgruppen bevorzugten deshalb bald Primaten wie Marmosets [31], Totenkopf- oder Rhesusaffen [32], weil deren Renin durch die 'massgeschneiderten' Humanrenin-Hemmer meist ähnlich stark blockiert wurde wie das menschliche. Diese Tiere sind aber nicht nur um ein Vielfaches aufwendiger in der artgerechten Haltung als Nager, sondern auch nicht in beliebiger Anzahl verfügbar. Zudem müssen sie vor den Versuchen kochsalzarm ernährt oder zur Ausscheidung des $\mathrm{NaCl}$ mit einem Diuretikum vorbehandelt werden. Dadurch wird der Blutdruck stark vom RAS abhängig. Eine Hemmung des Renins in diesem Zeitpunkt führt zu einer messbaren Blutdrucksenkung, obwohl die Tiere einen normalen Blutdruck aufweisen. Während der Versuche können bei grösseren Primaten auch Plasmaproben genommen und so die PRA gemessen werden. Dabei trat bei vielen Hemmern ein unerwarteter Effekt auf: Obwohl die PRA oft für viele Stunden praktisch vollständig gehemmt war, stieg der Blutdruck nach einer gewissen Zeit wieder auf den Kontrollwert vor dem Versuch an (s. Fig. 4). Dieser Befund hat die Frage nach einem für den Blutdruck relevanten 'Gewebe'-Renin aufgeworfen, das bis heute Gegenstand kontroverser Ansichten ist.

Mit dieser Methode haben wir an Totenkopfäffchen und Rhesusaffen über hundert Renin-Hemmer geprüft. Das Ergebnis ist leicht zusammenzufassen: Die meisten Verbindungen zeigen nach $i . v$. Applikation eine deutliche, mehr oder weniger lang andauernde PRA-Hemmung und meistens, aber nicht immer, auch eine Blutdrucksenkung. Aber nur mit Hemmem aus der 'nichtpeptidischen' Klasse wie z.B. SDZ 217-172 konnten reproduzierbare Effekte nach oraler Verabreichung (mittels Magensonde)

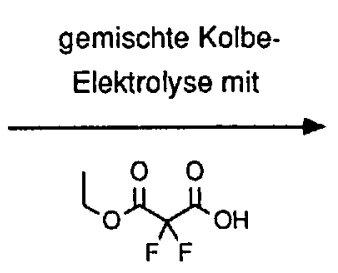

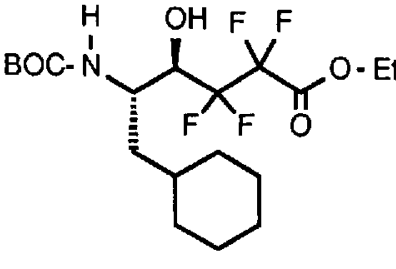

$15-20 \%$ 


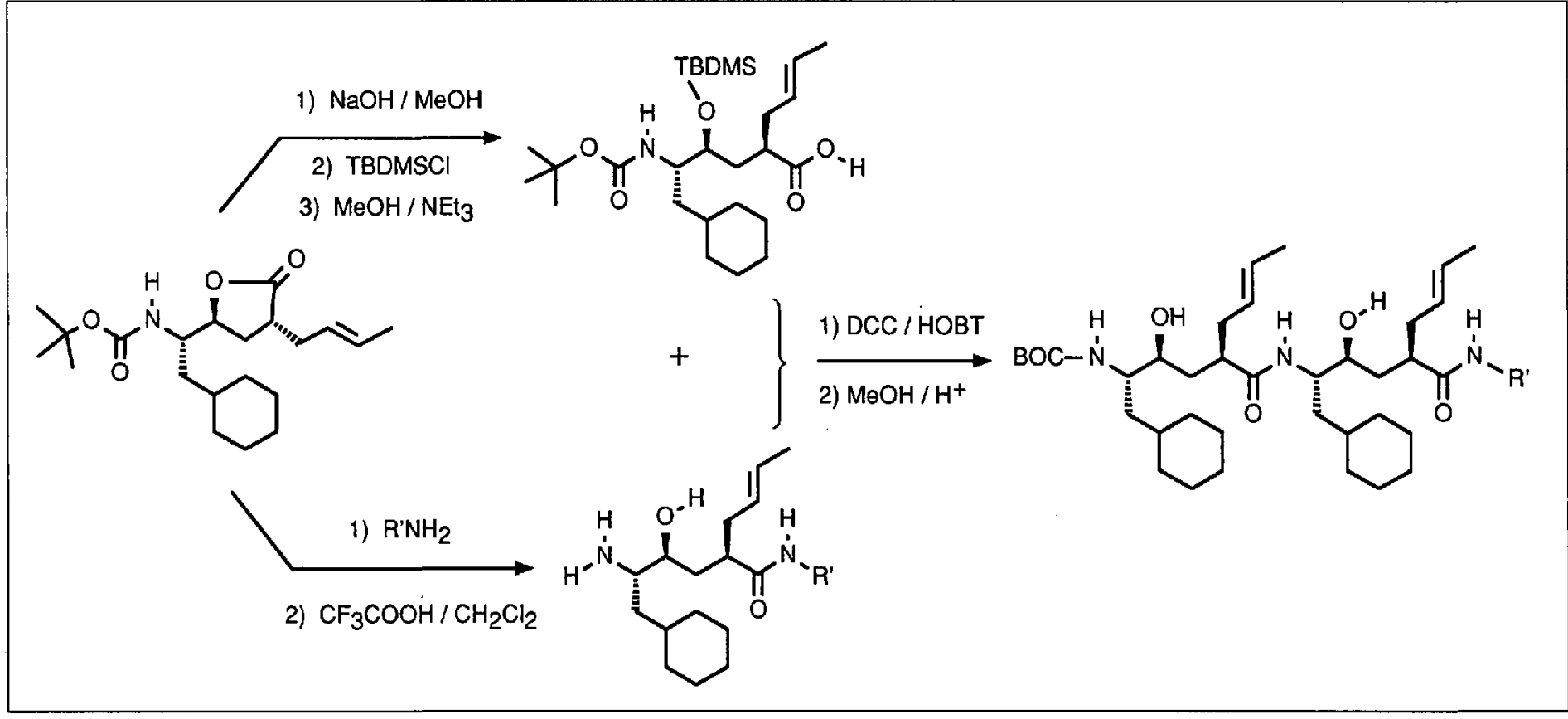

beobachtet werden. Wie aus der Gegenüberstellung in Fig. 4 ersichtlich ist, wird für die gleiche Wirkung aber immer noch rund die zwanzigfache Menge der intravenösen Dosis benötigt.

\section{Schlussfolgerungen}

Die jetzt schon rund ein Jahrzehnt dauernden Anstrengungen vieler Forschungsgruppen (zum grossen Teil aus der Pharmaindustrie), klinisch brauchbare Reninhemmer zu entwickeln, hat bis jetzt folgende Ergebnisse gebracht:

Es hat sich gezeigt, dass mit dem Konzept der 'transition state'- und Substratanalogen Proteasehemmer in kurzer Zeit äusserst potente und selektive 'Lead'-Verbindungen generiert werden können. Naturgemäss sind diese Substanzen aber peptidartige Verbindungen mit all den vorgängig beschriebenen Nachteilen. 'Gewöhnliche' Peptide sind in vielen Fällen ganz einfach zu 'soft' für eine breite, insbesondere orale Applikation. Diese Nachteile durch entsprechende chemische Modifikationen zu verbessern hat sich als die weit schwierigere Aufgabe herausgestellt, als die ersten Peptid-'Leads' zu finden.

Tatsächlich war bis jetzt eine orale Wirksamkeit an Primaten nur dann nachweisbar, wenn den Testsubstanzen der Peptidcharakter weitgehend fehlte, d.h. die Verbindungen enthielten keine oder höchstens noch eine $\alpha$-Aminosäure. Aber selbst dann beträgt die (Effekt)-Bioverfügbarkeit meist nur wenige Prozente. Die Tatsache, dass heute eigentlich kaum mehr eine grosse Nachfrage für neue antihypertensive Wirkstoffe besteht (ironischerweise nicht zuletzt wegen dem guten Wirkungsprofil der ACEHemmer!) hat in jüngster Zeit dazu geführt, dass die ambitiöse Suche nach oral wirksamen Renin-Hemmer vielerorts, so auch bei uns, reduziert worden ist.
Die erzielten grossen Forschritte in der Planung und Synthese von 'transition-state'-Renin-Hemmern waren jedoch keineswegs vergebens, selbst wenn einzelne Gruppen das ursprüngliche Ziel, neue antihypertensive Wirkstoffe mit dieser Strategie zu finden, aufgegeben haben. Die erlangten Erkenntnisse können mühelos auf andere Proteasen übertragen werden. Und Renin ist ja bei weitem nicht das einzige proteolytische Enzym, durch dessen Hemmung ein therapeutischer Nutzen erhofft werden kann. Das aktuellste Ziel auf diesem Gebiet ist sicher die HIV-Protease, ein vom AIDS-Virus codiertes Enzym, das für die virale Replikation unentbehrlich ist [37]. Da es wie Renin auch eine Asparyl-Protease ist, können die gleichen, in dieser Arbeit beschriebenen 'Rezepte' angewandt werden. Tatsächlich hat es sich gezeigt, dass viele ReninHemmer, obwohl nicht für die HIV-Protease optimiert, ausgezeichnete 'Lead'-Verbindungen als HIV-Blocker sind. Zudem kommen für eine antivirale Therapie auch Wirkstoffe in Betracht die zunächst nicht oral wirksam sind.

Dank gebührt meinen Mitarbeitern K. Christ und Ch. Rich, die die meisten der in dieser Arbeit erwähnten Verbindungen synthetisiert haben. All meinen Kollegen aus der Chemie, Biochemie und Pharmakologie, die am Renin-Projekt beteiligt waren, danke ich für die jahrelange, interessante und konstruktive Zusammenarbeit. Vor allem aber Dr. E. Pfenninger, dem 'Pionier' in Sachen Protease-Hemmer in unserer Firma!
Eingegangen am 14. Oktober 1991

[1] M. A. Ondetti, Circulation 1988, 77, 1-74

[2] a) B. Waeber, J. Nussberger, H. R. Brunner, in 'New Therapeutic Strategies in Hypertension', Eds. N. M. Kaplan, B. M. Brenner und J. H. Larags, Raven Press, New York, 1989, S. 97; b) J. I. S. Robertson, Drugs 1990, 40, 326.

[3] A.R. Sielecki, K. Hayakawa, M. Fujinaga, M. E. P. Murphy, M. Fraser, A. K. Muir, C. T. Carilli, J. A. Lewicki, J. D. Baxter, M. N. G. James, Science 1989, 243, 1346.

[4] D. H. Rich, in 'Proteinase Inhibitors', Eds. A. J. Barret und G. Salvesen, Elsevier, Amsterdam, 1986, S. 179-208.

[5] H. Umezawa, T. Aoyagi, H. Morishima, M. Matsuzaki, M. Hamada, T. Takeuchi, I. Antibiot. 1970, 23, 259

[6] J. Boger, N. S. Lohr, E. H. Ulm, M. Poe, E. H. Blaine, G. M. Fanelli, T. Y. Lin, L. S. Payne T. W. Schorn B. I. LaMont, T. C. Vassil, I. I. Stabilito, D. F. Veber, D. H. Rich A. S. Bopari, Nature (London) 1983, 303, 81.

[7] a) J. Burton, E. E. Slater, P. Corvol, J. Menard, L. H. Hartley, Clin. Exp. Hypertens. Theory Pract. 1982, A4, 322; b) F.X. Galen, C. Devaux, $S$. Altlas, T. Guyenne, J. Menard, P. Corvol, D. Simon, C. Cazaubon, P. Richer, G. Badouaille, J. P. Richaud, P. Gros, B. Pau,J. Clin. Invest. 1984, 74, 723; c) V.J. Dzau, R. I. Kopelman, A. C. Barger, E. Haber, Am. J. Physiol. 1984, 246, $\mathrm{H} 404$.

[8] R. J. Cody, J. Burton, G, Evin, K. Poulson, J. A. Herd, E. Haber, Biochem. Biophys. Res. Commun. 1980, 97, 230.

[9] J.M. Wood, C. Heusser, N. Gulati, P. Forgiarini, K. G. Hofbauer, Hypertension 1986, 8, 600 .

[10] B. Leckie, M. Szelke, B. Atrash, S. R. Battie, A. Hallett, D. M. Jones, G. D. McIntyre, J. Suerias, D. J. Webb, Biochem. Soc. Trans. 1985, 13, 1029.

[11] M. Szelke, D. M. Jones, B. Atrash, A. Hallet, B. J. Leckie, in 'Peptides, Structure and Function', Proceedings of the Eighth American Peptide Symposium, Eds. V.J. Hruby und D. H. Rich, Pierce Chemical Co., Rockford, Ill., 1983, S. 579.

[12] J.Boger,L. S. Payne, D. S. Perlow, N. S. Lohr, M. Poe, E. H. Blaine, E. H. Ulm, T.W. Schorn, B. I. LaMont, T. Y. Lin, M. Kawai, D. H. Rich, D. F. Veber, J. Med. Chem. 1985, 28, 1779.

[13] Für neuere Übersichten s. a) W. J. Greenlee Pharmac. Res. 1987, 4, 364; b) W. J. Greenlee. Med.Res. Rev. 1990, 10, 173; c) T. D. Ocain, M. Abou-Gharbia, Drugs Future 1991, 16, 37.

[14] J. R. Luly, J. J. Plattner, H. Stein, N. Yi, J. Soderquist, P. A. Marcotte, H. D. Kleinert, T. J. 
Perun, Biochem. Biophys. Res. Commun. 1987, $143,44$.

[15] a) B. Weidmann, Patent DE 87-3742474, 1987 b) B. Weidmann, Patent DE 3841319, 1989.

[16] J. J. Plattner, P. A. Marcotte, H. D. Kleinert, $J$ Med. Chem. 1988, 3I, 2277.

[17] a) S. Thaisrivongs, D. T. Pals, D. W. Harris, W M. Kati, S. R. Turner, J. Med. Chem. 1986, 29, 2088; b) G. J. Hanson, J. S. Baran, H. S. Lowrie, Biochem. Biophys. Res. Commun. 1989, 160,1.

[18] A. Vedani, J. Comput. Chem. 1986, 7, 701.

[19] A. Widmer, Interner Bericht, Sandoz AG, 1987.

[20] E.Pfenninger hat später eine Synthese des 'richtigen' Hydroxyethylen-Isosteren für Phe-His entwickelt. Persönliche Mitteilung, Sandoz, 1990.

[21] M. Bodanszky, A. Bodanszky, 'The Practice of Peptide Synthesis’, Springer-Verlag, Berlin, 1984 , S. 145.

[22] S. Thaisrivongs, D. T. Pals, W. M. Kati, S. R. Tuner, L. M. Thomasco, W. Watt, J.Med.Chem 1986, 29, 2080.

[23] J.C.Collins, W.W. Hess, F.J. Frank, Tetrahedron Lett. 1968, 3363.

[24] K. E. Pfitzner, J. G. Moffat, J. Am. Chem. Soc. $1965,87,5661$

[25] D. B. Dess, J. C. Martin, J. Org. Chem. 1983, 48, 4155.
[26] R. Henning, Nachr. Chem. Tech. Lab. 1990,38, 460.

[27] A. H. Fray, R. L.Kaye, E. F. Kleinman, J. Org. Chem. 1986, $51,4828$.

[28] E. Pfenninger, B. Weidmann, A. Stoll, Patent GB 86-19182, 1986.

[29] D. B. Darnon, D. J. Hoover, J. Am. Chem. Soc. $1990,112,6439$

[30] a) B. Weidmann, Patent DE 3815913, 1988; b) B. Weidmann, Patent WO 8910917 A2, 1989.

[31] J. M. Wood, N. Gulati, P. Forgiarini, W. Fuhrer, K. G. Hofbauer, Hypertension 1985, 7, 797.

[32] D. T. Pals, S. Thaisrivongs, J. A. Lawson, W. M Kati, S. R.Tuner, G. L. DeGraa, D. W. Harris, G. A. Johnson, Hypertension 1986, 8, 1105; M. DeClaviere, C. Cazaubon, C. Lacour, D. Msato J. P. Gagnol, G. Evin, P. Corvol, J. Cardiovasc. Pharmacol. 1985, 7 (Suppl. 4), 58; M. Tree, B. Atrash, B. Donovan, A. Hallett, M. Hughes, A. F. Lever, J. Morton, J. Sueiras-Dias, J. Hypertension 1985, 3, 13.

[33] J. R. Huff, J. Med. Chem. 1991, 34, 2305.

[34] a)F.Cumin, D.Le-Nguyen, B. Castro, J. Menard, P. Corvol, Biochem. Biophys. Acta 1987, 258, 413 ; b) F. Cumin, D. Nisato, J.-P. Ganol P. Corvol, J. Cardiovasc. Pharmacol. 1987, JO (Suppl. 7), 102.
[35] J. P. Evenou, B. Weidmann, E Pfenninger, R Metternich, H. Wagner, Biochem. Pharmacol 1990, 40, 765 .

[36] a) A. Hagenbach, R. Metternich, E. Pfenninger, B. Weidmann, Patent DE 87-3701526, 1987; b) A. Hagenbach, R. Metternich, E. Pfenninger, B Weidmann, Patent DE 3800591 Al: c) M. G Bock, R. M. DiParddo, B. E. Evans, R. M Freidinger, K. E. Rittle, L. S. Payne, J. Boger, W. L. Whitter, B. I. LaMont, E. H. Ulm, E.H. Blaine, T. W. Schorn, D. F. Veber, J. Med. Chem. 1988 31, 1918; d) G. L. Bundy, D. T. Pals, J. A. Lawson, S. J. couch, M. F. Lipton, M. A. Mauragis, ibid. 1990, 33, 2276

[37] R. H. Bradbury, J. S. Major, A. A. Oldham, J. E. Rivett, D. A. Roberts, A. M. Slater, D. Timms, D. Waterson, J. Med. Chem. 1990, 33, 2335.

[38] a) M.de Gasparo, F. Cumin, J. Nussberger, T.T Guyenne, J. M. Wood, J. Menard, Br. J. Clin Pharmacol. 1989, 27, 587; b) X. Jeunemaitre, J Menard, J. Nussberger, T. T. Guyenne, H. R. Brunner, P. Corvol, Am. J. Hypertens. 1989, 2 819.
Chimia 45 (1991) 376-378

(C) Schweiz. Chemiker-Verband; ISSN 0009-4293

\section{Bildung 12- bis 40-gliedriger Oligolide aus enantiomerenreinen 3-Hydroxybuttersäure-Derivaten - Bausteine für eine $2_{1}$ - und eine 3,-Helix}

\section{Hans-Martin Müller, Max Dobler, Peter Zbinden und Dieter Seebach*}

Abstract. Trimeric, tetrameric, and pentameric oligolides are formed preferentially from $(S)$ - $\beta$-butyrolactone, or from methyl $(R)$-3-hydroxybutanoate, upon prolonged treatment with catalytic amounts of tetraoxadistannacyclodecane (Shanzer's macrolactonization catalyst) in refluxing xylene. The crystal structure of the tetramer is described. Substructures of the larger folded oligolides are used for the construction of a left-handed $2_{1}$-helix, as found in the biopolymer PHB, and a heretofore unknown right-handed 3 -helix.

In zwei früheren Arbeiten [1] haben wir über die Isolierung von Oligoliden aus fünf bis sieben 3-Hydroxybuttersäure-Einheiten aus Makrolaktonisierungsansätzen nach Yamaguchi [2] und Shanzer [3] berichtet. Wir äusserten den Verdacht, dass Oligolide aus weniger als fünf Einheiten, die wir damals nicht entdeckten, sich eher aufgrund kinetischer als thermodynamischer Effekte nicht bilden

Wie wir jetzt fanden, werden nach den genannten Methoden mit ( $S$ )- $\beta$-Butyrolacton (1/ kat. 2) [4], (R)-3-Hydroxybuttersäure und dem zugehörigen Methylester [5]

*Korrespondenz: Prof. Dr. D. Seebach Laboratorium für Organische Chemie, Eidgenössischen Technischen Hochschule ETH-Zentrum

Universitätstrasse 16

CH-8092 Zürich

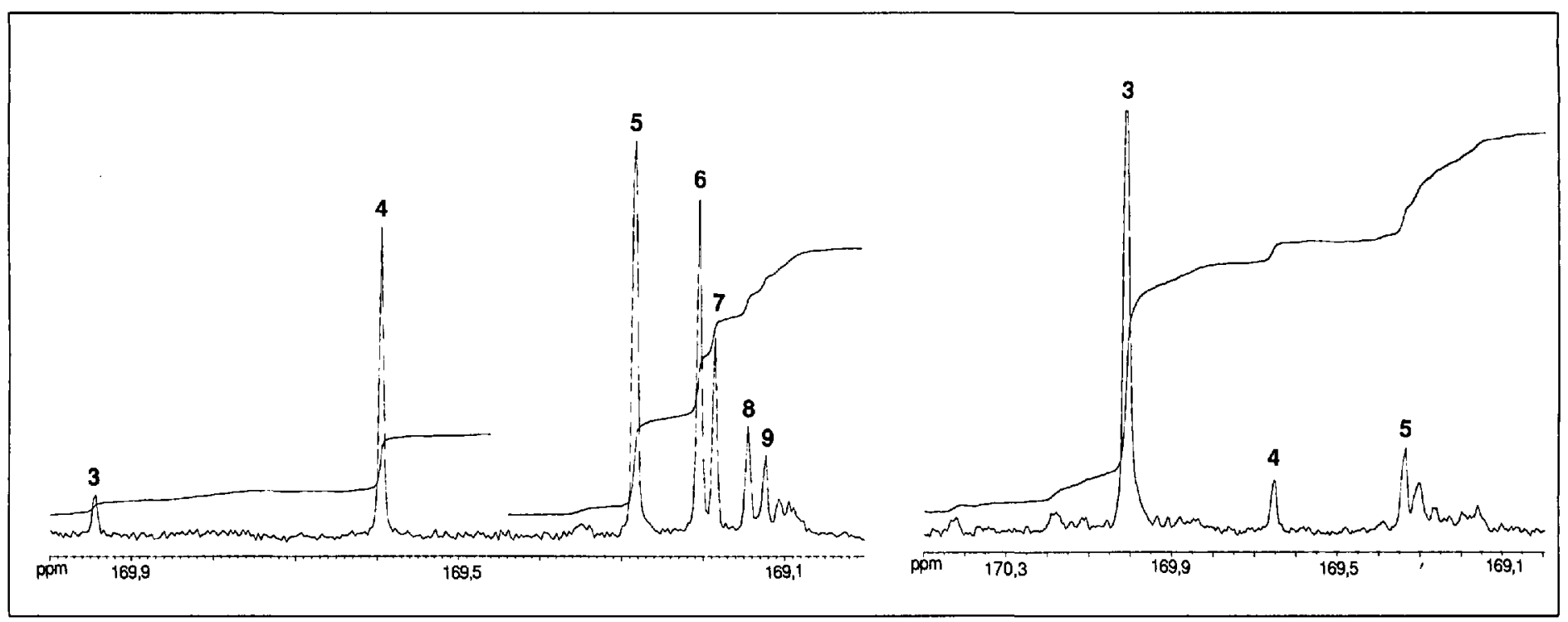

Fig. $1 .{ }^{13} \mathrm{C}$-NMR-Spektren $(100 \mathrm{MHz})$ der Carbonyl-Region von Oligolid-Gemischen. Messbedingungen: invers-gated-Experiment mit einer Relaxationsverzögerung von $3 \mathrm{~s}$, einer Aquisitionszeit von $3.2 \mathrm{~s}$ und einem Pulswinkel von $45^{\circ}$. Links: Rohprodukt des im Schema beschriebenen Ansatzes. Rechts: Rohprodukt einer analogen Reaktion in siedendem Xylol (114 h) statt Benzol. 